Internat. J. Math. \& Math. Sci.

Vol. 23, No. 1 (2000) 31-35

S0161171200001721

(C) Hindawi Publishing Corp.

\title{
ON THE EXTENSION OF POSITIVE LINEAR FUNCTIONALS
}

\author{
ERGASHBOY MUHAMADIEV and ADEL T. DIAB
}

(Received 12 September 1997 and in revised form 20 April 1998)

\begin{abstract}
A necessary and sufficient condition to extend a continuous linear real functionals which is positive with respect to a semi-group defined on a subspace of a linear space is discussed in this paper. The case of a closed subspace of a Banach space is also discussed.
\end{abstract}

Keywords and phrases. Solid semi-group, extension functional, positive functional, monotonic Banach functional.

2000 Mathematics Subject Classification. 46A22, 46A40.

1. Introduction. Let $E$ be a real linear space with a linear semi-group $K$ (we will call the set $K \subset E$ a linear semi-group if it possesses the following properties: $K+K \subset K$, and $\alpha \cdot K \subset K$ for all $\alpha \geq 0$ ) with $K \subset E$ (the word, linear will be omitted) and let $L \subset E$ be a subspace of $E$. Also, let $f_{0}$ be a real linear functional on $L$. A real linear functional $f$ on $E$ is called an extension of $f_{0}$ if $f(x)=f_{0}(x)$ for every $x \in L$. A linear functional $f$ will be called positive (with respect to $K$ ) if $f(x) \geq 0$ for every $x \in K$.

In the theory of spaces with an associated semi-group, the existence of the extension of positive linear functional plays an important role. Later on, several mathematicians studied a necessary condition to extend a linear continuous positive functional, for example, M. G. Krein theorem [4] states that "if $K \neq E$ is a semi-group in a Banach space $E$ with interior point and the subspace $L \subset E$ contains at least one interior point of $K$, then each linear continuous positive functional on $L$ (i.e., $f_{0}: L \rightarrow \mathbb{R}$ such that $f_{0}(x) \geq 0$ for every $\left.x \in K \cap L=K_{L}\right)$ can be extended to a linear continuous positive functional $f$ defined on $E$ (i.e., $f: E \rightarrow \mathbb{R}$ such that $f_{0}(x)=f(x)$ for every $x \in L$, and $f(x) \geq 0$ for every $x \in K)$." Other important theorems on the existence of positive extension of linear functionals are given in $[1,2,3]$.

2. Main results. In the present work, we introduce a new condition for the existence of the extension of positive linear functionals which depend upon the definition of Banach functional [5]. Two cases will be considered separately.

CASE (A). $E$ is a real linear space with a semi-group $K$.

The functional $\omega: E \rightarrow \mathbb{R}$ is called a Banach functional if

(1) $\omega(x+y) \leq \omega(x)+\omega(y)$ for every $x, y \in E$,

(2) $\omega(\lambda \cdot x)=\lambda \cdot \omega(x)$ for every $x \in E$, for every $\lambda \geq 0$.

We say that the Banach functional $\omega$ is monotonic (with respect to $K$ ), if $\omega(x+y) \geq$ $\omega(x)$ for every $x \in E$ and for every $y \in K$.

The following theorem is close to [1, Thm. 2.3]. 
THEOREM 2.1. Let $L \subset E$ be a subspace of a linear space $E$, and $f_{0}: L \rightarrow \mathbb{R}$ be a linear functional such that $f_{0}(x) \geq 0$ for every $x \in K_{L}$. Then a necessary and sufficient condition for the existence of a linear functional $f: E \rightarrow \mathbb{R}$, such that $f_{0}(x)=f(x)$ for every $x \in L$ and $f(x) \geq 0$ for every $x \in K$, is the existence of a monotonic Banach functional $\omega$ satisfying the inequality

$$
f_{0}(x) \leq \omega(x) \text { for every } x \in L .
$$

Proof. Let the functional $f$ be an extension of $f_{0}$. We define the functional $\omega$ by $\omega(x)=\inf \{|f(x+y)|: y \in K\}$, where $x \in E$. Obviously, $\omega$ is a monotonic Banach functional which satisfies inequality (2.1). (Another proof, $f$ is already a monotonic Banach functional and hence we take $\omega=f$.)

Conversely, consider the existence of a monotonic Banach functional $\omega$ satisfying inequality (2.1). Then by the Hahn-Banach theorem there exists a linear functional $f$ on $E$ such that $f_{0}(x)=f(x)$ for every $x \in L$ and $f(x) \leq \omega(x)$ for every $x \in E$. It remains to prove that $f$ is positive. Let $x \in K$, then from the fact that $\omega$ is monotonic, we get $\omega(-x) \leq 0$ and therefore $f(-x) \leq \omega(-x) \leq 0$, which implies that $f(x) \geq 0$. Hence $f$ is a positive extended functional of $f_{0}$. This completes the proof of the theorem.

In the following theorem, the condition that the Banach functional is monotonic is dropped.

THEOREM 2.2. Let $L \subset E$ be a subspace of a linear space $E$, $\varpi$ be a Banach functional (not necessarily monotonic), and $f_{0}: L \rightarrow \mathbb{R}$ be a linear functional such that

$$
f_{0}(x) \leq \varpi(x+y) \quad \text { for every } x \in L, \text { for every } y \in K .
$$

Then $f_{0}$ has an extension functional $f$ on $E$ such that $f(x) \geq 0$ for every $x \in K$.

Proof. Let $\omega(x)=\inf \{\varpi(x+y): y \in K\}$ for every $x \in E$. We start by showing that

(i) $\omega(x)>-\infty$ for every $x \in E$,

(ii) $f_{0}(x) \leq \omega(x)$ for every $x \in L$,

(iii) $\omega$ is a monotonic Banach functional.

Condition (i) is a consequence of inequality (2.2) for, if $x=0$, then $\varpi(y) \geq 0$ for every $y \in K$, but $\varpi$ is a Banach functional, then $0 \leq \varpi(y)=\varpi(x+y+(-x)) \leq$ $\varpi(x+y)+\varpi(-x)$, which implies that $\varpi(x+y) \geq-\varpi(-x)$ for every $y \in K$ and for every $x \in E$. Therefore, $\omega(x) \geq-\varpi(-x)>-\infty$ for every $x \in E$, this proves condition (i). Since $f_{0}(x) \leq \varpi(x+y)$ for every $x \in L$, every $y \in K$, then $f_{0}(x) \leq \omega(x)$ for every $x \in E$. This proves condition (ii).

Clearly $\omega$ is a Banach functional and it remains to show that $\omega$ is a monotonic functional (i.e., $\omega(x) \leq \omega(x+y)$ for every $y \in K$ and every $x \in E)$. Since $\omega(x+y)=$ $\inf \left\{\varpi\left(x+y+y_{1}\right): y_{1} \in K\right\}=\inf \left\{\varpi(x+z): z=y+y_{1} \in K, y_{1} \in K\right\} \geq \inf \{\varpi(x+$ $u): u \in K\}=\omega(x)$ for every $y \in K$ and every $x \in E$, and hence condition (iii) is satisfied. Moreover, by using Theorem 2.1, there exists an extension functional $f$ of the functional $f_{0}$ such that $f(x) \geq 0$ for every $x \in K$. This completes the proof of the theorem. 
CASE (B). $E$ is a Banach space with closed semi-group $K$.

We introduce the following definition.

Definition. We define the following functional $|x|_{K}=\inf \{\|x+y\|: y \in K\}$ for each $x \in E$. Obviously, $|\cdot|_{K}$ is a monotonic functional with respect to the semi-group $K$ and $|\cdot|_{K}$ is a Banach functional, moreover, $|x|_{K} \leq\|x\|$ for every $x \in E$ (hence the functional $|\cdot|_{K}$ is a continuous functional on $E$ ). Also, from the closeness of a semigroup $K$, we have $|x|_{K}=0$ if and only if $-x \in K$.

EXAMPLE 1. Let $E=\ell_{2}=\left\{x=\left(\xi_{i}\right): \sum_{i}\left|\xi_{i}\right|^{2}<\infty\right\}, K=\left\{\left(\xi_{i}\right) \in \ell_{2}: \xi_{i} \geq 0, i=1,2, \ldots\right\}$. Obviously, for every $x=\left(\xi_{i}\right) \in \ell_{2}, x=x_{+}-x_{-}$, where $x_{+}=1 / 2\left(\left|\xi_{i}\right|+\xi_{i}\right)$, $x_{-}=1 / 2\left(\left|\xi_{i}\right|-\xi_{i}\right)$, then $x_{+}, x_{-} \in K$ and $|x|_{K}=\left\|x_{+}\right\|$.

The following theorem is analogous to Theorem 2.1.

THEOREM 2.3. Let $L$ be a closed subspace of a Banach space $E$ with a closed semigroup $K$, and let $f_{0}: L \rightarrow \mathbb{R}$ be a linear continuous functional such that $f_{0}(x) \geq 0$ for every $x \in K_{L}$. Then a necessary and sufficient condition that there exists a linear continuous functional $f: E \rightarrow \mathbb{R}$ such that $f_{0}(x)=f(x)$ for every $x \in L$ and $f(x) \geq 0$ for every $x \in K$ is that there exist numbers $\alpha>0, \beta>0$ and a monotonic Banach functional $\omega$ that satisfies the inequalities

$$
\begin{gathered}
f_{0}(x) \leq \alpha \cdot \omega(x) \quad \text { for every } x \in L, \\
\omega(x) \leq \beta \cdot\|x\| \text { for every } x \in L .
\end{gathered}
$$

PRoof. For the proof of the theorem, we need the following lemma.

LEMmA 2.1. The linear continuous functional $f$ is positive with respect to a semigroup $K$ if and only if there exists a number $C>0$ such that

$$
f(x) \leq C \cdot|x|_{K} \quad \text { for every } x \in E .
$$

Proof. If $f$ is positive, then $f(y) \geq 0$ for every $y \in K$. Let $x \in E$, then $x \leq x+y$ for every $y \in K$. This implies that $f(x+y)=f(x)+f(y) \geq f(x)$ and therefore $f(x) \leq f(x+y) \leq|f(x+y)| \leq\|f\|\|x+y\|$. By taking the infimum of both sides of the previous inequality, we get $C=\|f\|, f(x) \leq C \cdot|x|_{K}$. Conversely, let $f$ be a linear continuous functional and there exists a number $C>0$ such that $f(x) \leq C \cdot|x|_{K}$ for every $x \in E$. In particular if $y \in K$, then $-y \in-K$ and we obtain that $-f(y)=$ $f(-y) \leq C|-y|_{K}=0$. Hence, $f(y) \geq 0$ for every $y \in K$.

Now we return to the proof of Theorem 2.3.

Let the linear continuous functional $f_{0}$ on $L$ has a linear continuous positive extension functional $f$ on $E$. Then by Lemma 2.1, there exists a number $C>0$ such that $f(x) \leq C \cdot|x|_{K}$ for every $x \in E$. Put $\omega(x)=|x|_{K}, \alpha=C$ and $\beta=1$. Hence $f_{0}(x) \leq C \cdot \omega(x)$ and $\omega(x) \leq\|x\|$ for every $x \in L$. Conversely, let $f_{0}$ be a continuous linear functional on $L$ which satisfies inequalities (2.3) and (2.4). Hence by the Hahn-Banach theorem there exists an extension functional $f$ of $f_{0}$ on $E$ such that $f(x) \leq \alpha \cdot \omega(x)$ for every $x \in E$. Hence from the fact that $\omega$ is monotonic, we obtain that $f(x) \geq 0$ for every $x \in K$, and from the following inequality:

$$
f(x) \leq \alpha \cdot \omega(x) \leq \alpha \cdot \beta \cdot\|x\|,
$$


we obtain that $f$ is a linear continuous functional on $E$. This completes the proof of the theorem.

COROLLARY 2.1. The linear continuous positive functional $f_{0}$ on a closed subspace $L$ of a Banach space $E$ has a linear continuous positive extension functional $f$ on $E$ if and only if there exists a number $C>0$ such that

$$
f_{0}(x) \leq C \cdot|x|_{K} \quad \text { for every } x \in L .
$$

Proof. This follows directly from Theorem 2.3.

In the following section, we discuss the relationship between the two functionals $|x|_{K}$ and $|x|_{K_{L}}$, where $|x|_{K_{L}}=\inf \left\{\|x+y\|: y \in K_{L}\right\}$ for each $x \in L$.

It is clear that $|x|_{K} \leq|x|_{K_{L}}$ for every $x \in L$. From Lemma 2.1, if $f_{0}$ is a linear continuous functional on a closed subspace $L$ in a Banach space $E$ such that $f_{0}(x) \geq 0$ for every $x \in K_{L}$, then there exists a number $C>0$ such that $f_{0}(x) \leq C \cdot|x|_{K_{L}}$ for every $x \in L$.

THEOREM 2.4. Given $C>0$ such that $|x|_{K_{L}} \leq C \cdot|x|_{K}$ for every $x \in L$. Then every continuous linear positive (with respect to a closed semi-group $K_{L}$ ) functional $f_{0}$ on $a$ closed subspace $L$ has a linear continuous positive (with respect to a closed semi-group $K)$ extension functional $f$ on $E$.

PROoF. The proof follows directly from Lemma 2.1 and Corollary 2.1.

We have to note that M. G. Krein theorem can be obtained directly from Theorem 2.4 and the following lemma.

LEMMA 2.2. Let $K \neq E$ be a semi-group in a Banach space $E$ with interior point (i.e., $K$ is a solid semi-group), and let the closed subspace $L \subset E$ contains at least one interior point of $K$. Then there exists a number $C>0$ such that $|x|_{K_{L}} \leq C \cdot|x|_{K}$ for every $x \in L$.

Proof. Let $u_{0} \in L$ be an interior point of $K$, then there exists $\delta_{0}>0$ such that $T\left(u_{0}, \delta_{0}\right) \subset K$, where $T\left(u_{0}, \delta_{0}\right)=\left\{u:\left\|u-u_{0}\right\| \leq \delta_{0}\right\}$. It is well known that $u_{\alpha}=$ $\alpha \cdot u_{0}+(1-\alpha) . y, 0<\alpha<1$ are the interior points of $K$ with radius $\alpha \cdot \delta_{0}$ (i.e., $T\left(u_{\alpha}\right.$, $\left.\alpha \cdot \delta_{0}\right) \subset K$ ) for every $y \in K$. Now, we suppose that $x \in L$ and $\|x\|=1$ then $Z_{\alpha}=$ $\left(\alpha \cdot u_{0}-(1-\alpha) \cdot x\right) \in L$ and $\left\|Z_{\alpha}-u_{\alpha}\right\|=(1-\alpha)\|x+y\|$. Therefore, if we choose $\alpha=\|x+y\| \cdot\left(\delta_{0}+\|x+y\|\right)^{-1}$, then $Z_{\alpha} \in K$ and

$$
\begin{aligned}
|x|_{K_{L}} & \leq\left\|x+Z_{\alpha}\right\|=\alpha \cdot \mid u_{0}+x \| \\
& \leq\left(\left\|u_{0}\right\|+1\right) \cdot\left(\delta_{0}+\|x+y\|\right)^{-1}\|x+y\| \\
& \leq\left(\left\|u_{0}\right\|+1\right) \delta_{0}^{-1}\|x+y\| \\
& =C \cdot\|x+y\|,
\end{aligned}
$$

where $C=\left(\delta_{0}\right)^{-1}\left(\left\|u_{o}\right\|+1\right)$. By taking the infimum of both sides of the last inequality at $y \in K$, we get

$$
|x|_{K_{L}} \leq C \cdot|x|_{K} \quad \text { for every } x \in L \text {. }
$$


The following example shows that, if the semi-group $K$ does not contain an interior point, then M. G. Krein theorem cannot be applied but our work can be applied.

EXAMPLE 2. Back to Example 1, it is known that $K$ is not solid semi-group in $E=\ell_{2}$. If $x_{1}=(1,1 / 2, \ldots, 1 / n, \ldots), x_{2}=\left(1,1 / 2^{2}, \ldots, 1 / n^{2}, \ldots\right)$, and $L=\left\{\alpha x_{1}+\beta x_{2}: \alpha, \beta \in \mathbb{R}\right\}$ is a subspace of $E$, then $K_{L}=K \cap L=\left\{\lambda x_{1}+\mu x_{2}: \lambda \geq 0, \lambda+\mu \geq 0\right\}$. Let $f_{0}: L \rightarrow \mathbb{R}$ be defined by $f_{0}(x)=f_{0}\left(\alpha x_{1}+\beta x_{2}\right)=\alpha C_{1}+\beta C_{2}$, where $C_{1}$ and $C_{2}$ are real numbers. Then if $C_{1}-C_{2}>0, C_{2}>0$, the functional $f_{0}$ satisfies inequality (2.7). Therefore, from Corollary 2.1, the linear continuous positive functional $f_{0}$ on $L$ has a linear continuous positive extension functional $f$ on $E$. We remark here that if $C_{2}=0$ and $C_{1}>0$, then clearly, the positive functional $f_{0}$ has no positive functional extension on $E$.

\section{REFERENCES}

[1] C. D. Aliprantis and O. Burkinshaw, Positive Operators, Pure and Applied Mathematics, vol. 119, Academic Press, Inc., Orlando, 1985. MR 87h:47086. Zbl 608.47039.

[2] R. E. Edwards, Functional Analysis. Theory and Applications, Holt, Rinehart and Winston, New York, Toronto, London, 1965. MR 36\#4308. Zbl 182.16101.

[3] M. A. Krasnosel'skij, Je. A. Lifshits, and A. V. Sobolev, Positive Linear Systems: The Method of Positive Operators, Sigma Series in Applied Mathematics, vol. 5, Heldermann Verlag, Berlin, 1989. MR 91f:47051. Zbl 674.47036.

[4] M. G. Krein and M. A. Rutman, Linear operators leaving invariant a cone in a Banach space, Trans. Amer. Math. Soc. (1950), no. 26, 128. MR 12,341b.

[5] V. Pta'k, On a theorem of Mazur and Orlicz, Studia Math. 15 (1956), 365-366. MR 18,320b. Zbl 071.10801.

Muhamadiev: Mathematical and Informational Department, Khujand's SCIENTIFIC CENTER, ACADEMY OF SCIENCES, KHUJAND, P.O. 3 BOX 113, TAJIKISTAN

E-mail address: muhamerg@khj.td.silk.org, math@scmd.khj.td.silk.org

Diab: Mathematics Department, Faculty of Science, Ain Shams University, Cairo, EGYPT

E-mail address: atdiab@frcu.eun.eg 


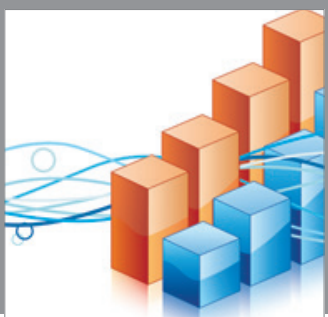

Advances in

Operations Research

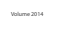

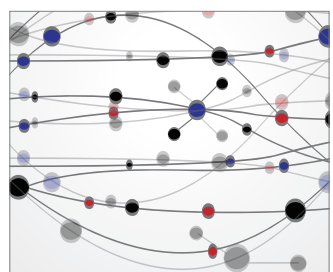

\section{The Scientific} World Journal
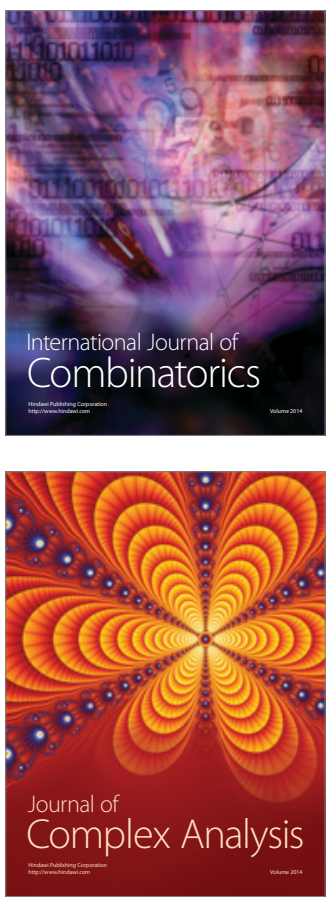

International Journal of

Mathematics and

Mathematical

Sciences
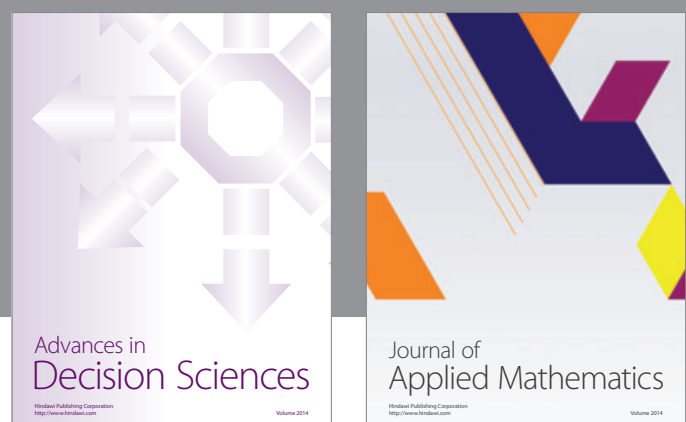

Journal of

Applied Mathematics
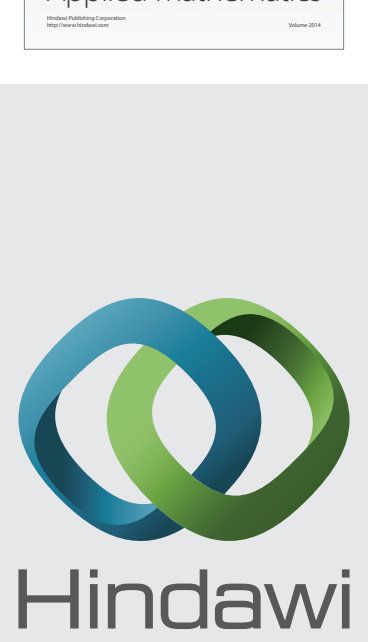

Submit your manuscripts at http://www.hindawi.com
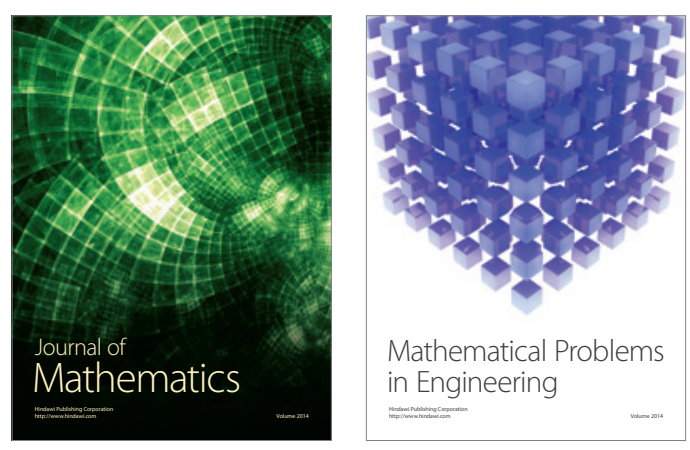

Mathematical Problems in Engineering
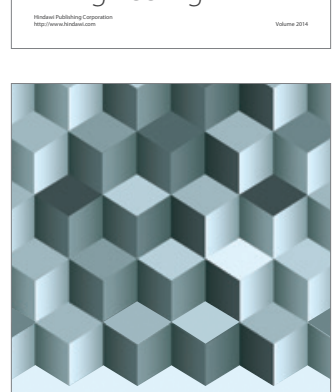

Journal of

Function Spaces
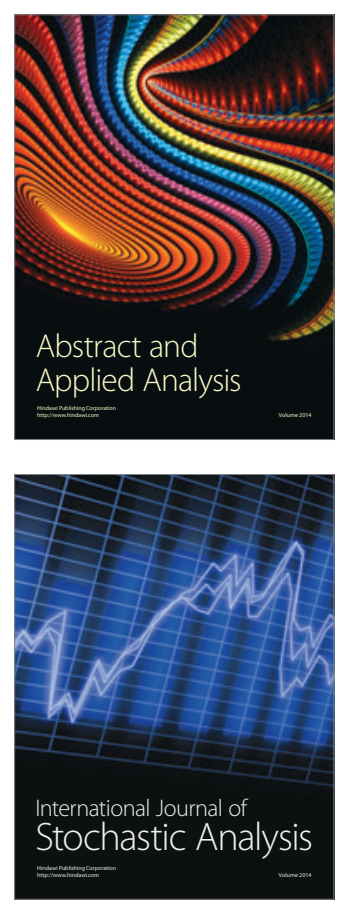

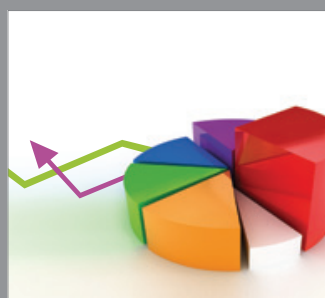

ournal of

Probability and Statistics

Promensencen
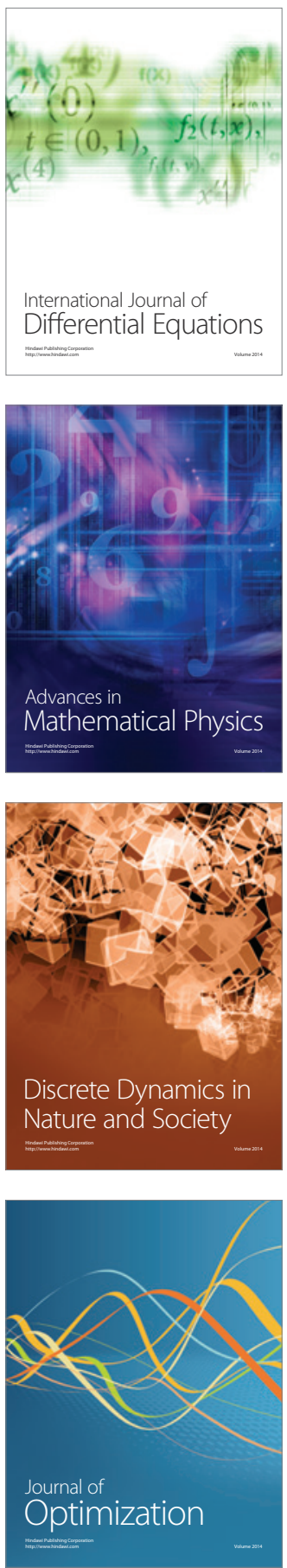